\title{
Extensão universitária como \\ ferramenta de promoção de saúde e ressignificação do cuidado à criança hospitalizada
}

\author{
Claudio José dos Santos Júnior \\ claudiosantos_al@hotmail.com \\ Jailton Rocha Misael \\ jailton.enf@gmail.com
}

Raquel de Lima Chicuta

raquellc1811@gmail.com

Maria Rosa da Silva

enfamariarosa@yahoo.com.br

Claudio Fernando Rodrigues Soriano

claudiofrsoriano@yahoo.com.br

John Victor dos Santos Silva

john.setedejulho@gmail.com

Universidade Estadual de Ciências da Saúde de Alagoas | Brasil

\section{Resumo}

Este trabalho descreve a experiência das ações desenvolvidas pelo Projeto de Extensão Universitária "Acolher", da Universidade Estadual de Ciências da Saúde de Alagoas (Uncisal), durante o ano de 2016. As intervenções realizadas foram pautadas no desenvolvimento de atividades lúdicoeducativas na área de saúde pediátrica nas Enfermarias de cuidados infantis do Hospital Geral do Estado, em Maceió-AL, através do emprego da ludoterapia e da educação em saúde. A partir da introdução de tais estratégias, pode-se propor alternativas às privações e restrições impostas pelo ambiente hospitalar, diminuindo a tensão do processo de internação e colocando em prática a promoção de saúde através de momentos de descontração, integração e de compartilhamento de conhecimentos.

\section{Palavras-chave}

Extensão Universitária; Educação em Saúde; Ludoterapia. 


\section{Introdução}

A hospitalização é uma condição de forte impacto na vida da criança e na rotina de todos que estão envolvidos diretamente com o seu cuidado infantil. Atividades como convívio escolar, lazer, relações familiares e sociais são prejudicadas pelo marcante processo de adaptação a realidade da internação. Ao ser afastada do seu ambiente domiciliar, atividades básicas diárias, como vestimentas, banho e alimentação, tornamse totalmente dependentes da rotina institucional e submetidas às restrições do contexto hospitalar.

Diversos autores discutem as modificações decorrentes desse processo. Saggese e Maciel (1997) trazem que a vida da criança é alterada durante a internação em um hospital, pois, para além do sofrimento com a doença, ela é afastada da família, da escola e dos objetos pessoais, perdendo grande parte de suas referências. Os autores trazem que encontramos nas unidades hospitalares um ambiente hostil, com procedimentos invasivos e uma atmosfera geralmente tensa e ameaçadora.

Angerami-Camon (1995) considera que, diante desse cenário, a criança pode entrar em um nível de sofrimento emocional e físico, podendo tais situações ser expressas através do medo de médicos, choro, agressividade, dependência, ansiedade, depressão, distúrbio do sono, evitação dos procedimentos médicos e outras formas de respostas.

Contribuem para esse processo de estranhamento e inadaptação da criança ao hospital a falta de variedade, de estrutura e de atividades para a interação da criança. Tudo isso dificulta sua adaptação no contexto hospitalar (GUIMARÃES, 1988).

Em contraposição, a humanização, conceituada por Mitre et al. (2004) como sendo "um fenômeno complementar e indispensável na assistência em que se valorizar a ética do cuidado e o reconhecimento das individualidades", se mostra um processo eficiente de minimizar os efeitos da hospitalização infantil.

Cruz, Costa e Nobrega (2006) veem a criança, enquanto ser especial e vulnerável a mudanças. Para os autores, esse público deve ter respeitadas suas necessidades em relação à hospitalização, bem como ter garantido o direito de receber uma assistência humanizada que promova a continuidade do seu crescimento e desenvolvimento, reduzindo os traumas provocados pela hospitalização. 
Extensão universitária como ferramenta de promoção de saúde

Corroborando com tais premissas, Vygotski (1991), a partir de seus estudos acerca da formação social da mente chegou à conclusão que as atividades lúdicas têm a função de facilitar o desenvolvimento integral da criança e o progresso de cada uma de suas funções psicológicas, intelectuais, motoras e morais.

Segundo Luchetti, Moreale e Parro (2011), o lúdico é um elemento essencial no trabalho com a criança, sendo uma estratégia de eficiente aplicação como recurso de comunicação na vinculação de informações de saúde.

Assim, nesse trabalho, parte-se da premissa de que a ludoterapia, aliada a humanização e a educação em saúde, surgem como estratégias de transmitir conteúdos educacionais e terapêuticos com a finalidade de estimular os usuários dos serviços a serem protagonistas do seu próprio cuidado em saúde através do prazer, da brincadeira e da alegria, tornando o processo de cuidado e de recuperação mais prazeroso e modificando a rotina triste e inquietante característica do hospital.

Nessa perspectiva, objetiva-se fazer relatar a experiência das ações e fazer reflexões acerca das vivências proporcionadas pelo Projeto de Extensão Universitária "Acolher", da Universidade Estadual de Ciências da Saúde de Alagoas (Uncisal), que tem como principal foco o desenvolvimento de atividades educativas na área de saúde pediátrica com pacientes internos e seus familiares, através do emprego da ludoterapia e da educação em saúde.

\section{Procedimento metodológico}

O presente trabalho configura-se como um estudo exploratório, do tipo relato de experiência. Seu objetivo é descrever as vivências oportunizadas pelas práticas do Projeto de Extensão Universitária "Acolher", integrante do Programa de Extensão "PróCriança" da Universidade Estadual de Ciência da Saúde de Alagoas (Uncisal), durante o ano de 2016.

A metodologia adotada foi a de pesquisa-ação com abordagem qualitativa fenomenológica, sendo que para sua construção foram empregadas as reflexões dos membros do Projeto acerca das ações por eles experimentadas e utilizados registros escritos e fotográficos extraídos do "diário de bordo" do Projeto. 
O referencial teórico utilizado foi obtido a partir de pesquisa bibliográfica em artigos científicos artigos indexados nas bases de dados SciELO e Google Acadêmico sobre as temáticas "educação em saúde", "ludoterapia” e "humanização".

De acordo com as orientações dos Periódicos Eletrônicos de Psicologia (2014), o gênero textual relato de experiência possui relevância devido a sua pertinência, importância dos problemas nele expostos e ainda devido ao nível de generalização na aplicação de procedimentos ou de resultados de intervenções em outras situações similares, ou seja, serve como colaboração à práxis metodológica de uma área do saber.

Segundo Gil (2008) a pesquisa-ação tem base empírica, apoia-se nas experiências vividas e na observação dos pesquisadores, e geralmente está relacionada com a solução de um problema de forma coletiva e participativa, no qual os pesquisadores estão envolvidos diretamente e de modo cooperativo ou participativo. Já o método fenomenológico, à luz de Merleau-Ponty (1999), é aplicado para o exame das experiências vividas e dos significados a elas atribuídos, e tem como etapas a descrição, a redução e compreensão dessas experiências, sendo que nessa última etapa ocorre a fase das interpretações.

\section{Resultados e Discussão}

O Projeto Acolher vem desenvolvendo suas atividades desde 2012 e conta com a participação de estudantes universitários para levar ações de educação em saúde para a rotina da Unidade Pediátrica do Hospital Geral do Estado Dr. Oswaldo Brandão Vilela HGE, localizado em Maceió (AL). A ação é cadastrada na Pró-Reitoria de Extensão da Uncisal (PROEX) e atualmente conta com 51 (cinquenta e um) voluntários que desenvolvem suas atividades sempre durante a semana, de segunda a sexta-feira, seguindo a um cronograma pré-estabelecido pela coordenação do Projeto.

O objetivo central do Acolher é levar a educação em saúde para o público infantil e seus acompanhantes por meio de estratégias lúdicas. Seus objetivos específicos incluem diminuir a tensão do ambiente hospitalar, proporcionando ao paciente e seus familiares momentos de integração e de compartilhamento de conhecimentos relativos à saúde pediátrica e aos direitos do público infantil; prezar pela qualidade de vida da criança durante o enfrentamento dos processos de adoecimento e internação; estimular o trabalho interdisciplinar e multiprofissional; e possibilitar aos discentes da área de 
Extensão universitária como ferramenta de promoção de saúde

saúde o desenvolvimento e a prática de habilidades de relacionamento interpessoal e humano.

Para ingresso no Projeto são realizadas seleções anuais abertas ao público universitário dos cursos de Enfermagem, Fonoaudiologia, Fisioterapia, Terapia Ocupacional, Medicina, Radiologia e áreas correlatas. O processo seletivo é composto por análise de disponibilidade do acadêmico, apreciação do currículo, avaliação da carta de intenção, e entrevista presencial. Todas as etapas tem como finalidade o recrutamento de integrantes que possuam o máximo das características necessárias ao desenvolvimento das atividades de campo, sendo que, são valorizados os candidatos comprometidos com o processo seletivo, que demonstrem criatividade, responsabilidade, empatia, apreço pelo outro e pela prática de características humanísticas, e os indivíduos capazes de reconhecer o ser humano como um todo e de valorizar a importância do cuidado integral, da relação humanizada e das suas potencialidades.

Os discentes selecionados passam a ser monitores de extensão e, ao longo do ano, participam de capacitações e oficinas de planejamento e de aprimoramento de habilidades, ambas visando o desenvolvimento de atitudes importantes e necessárias a abordagem dos pacientes durante as práticas; nesses momentos, objetiva-se ainda a qualificação e a apropriação de conceitos por parte dos integrantes sobre as temáticas a serem abordadas durante as visitas. Regularmente são realizadas reuniões com a finalidade de compartilhar as experiências oportunizadas pelos grupos e visando avaliar as atividades.

0 Acolher conta atualmente com doze grupos, que, distribuídos em dias diferentes da semana, desenvolvem suas atividades nos turnos da manhã e da tarde, seguindo o planejamento de temas (Tabela 1) programado em calendário. Cada grupo conta com uma equipe que varia de 3 a 6 acadêmicos, em que um deles, geralmente o mais antigo no Projeto, assume o papel de facilitador das ações. As equipes são multidisciplinares e preza-se pela integração das áreas conhecimento como forma de estímulo à abordagem multidisciplinar entre os futuros profissionais e vislumbrando uma visão ampliada do cuidado. 
Extensão universitária como ferramenta de promoção de saúde

\begin{tabular}{l} 
Temas \\
\hline Acidentes domésticos \\
Afogamento \\
Bullying \\
Direitos dos usuários do SUS \\
Estatuto da criança e do adolescente \\
Higiene bucal \\
Higiene dos alimentos \\
Alimentação saudável \\
Intoxicação \\
Lavagem das mãos e higiene pessoal \\
Prevenção de acidentes de trânsito \\
Queimaduras \\
Violência
\end{tabular}

Tabela 1: Planejamento de temas abordados durante as visitas do Projeto de Extensão Acolher em 2016. Fonte: Elaboração própria (2017).

Como mencionado, o foco do Acolher é o público infantil. Assim, suas intervenções são pautadas na aplicação de estratégias lúdicas visando à criação de um ambiente favorável ao enfrentamento da doença, da situação de hospitalização e à promoção da educação em saúde. Os materiais utilizados nas visitas (manuais explicativos, álbuns de imagens, folders e jogos temáticos) são elaborados pelos próprios monitores em oficinas temáticas executadas pela coordenação do Projeto. Durante a fase de elaboração desses instrumentos prioriza-se o emprego de uma linguagem apropriada a fim de facilitar a compreensão das informações pelo público atendido. 


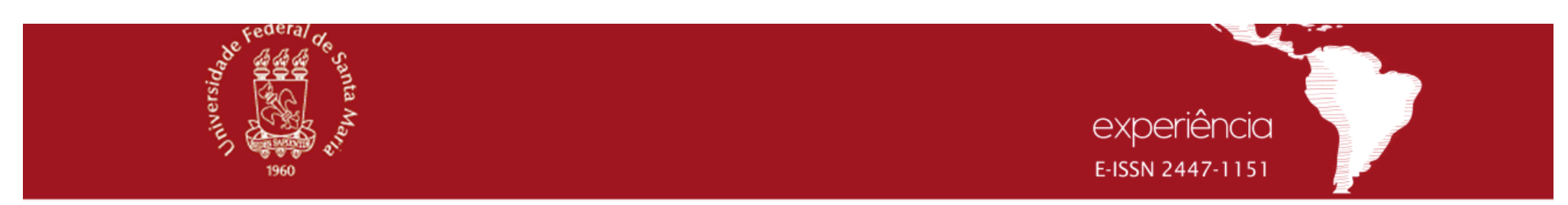

Extensão universitária como ferramenta de promoção de saúde

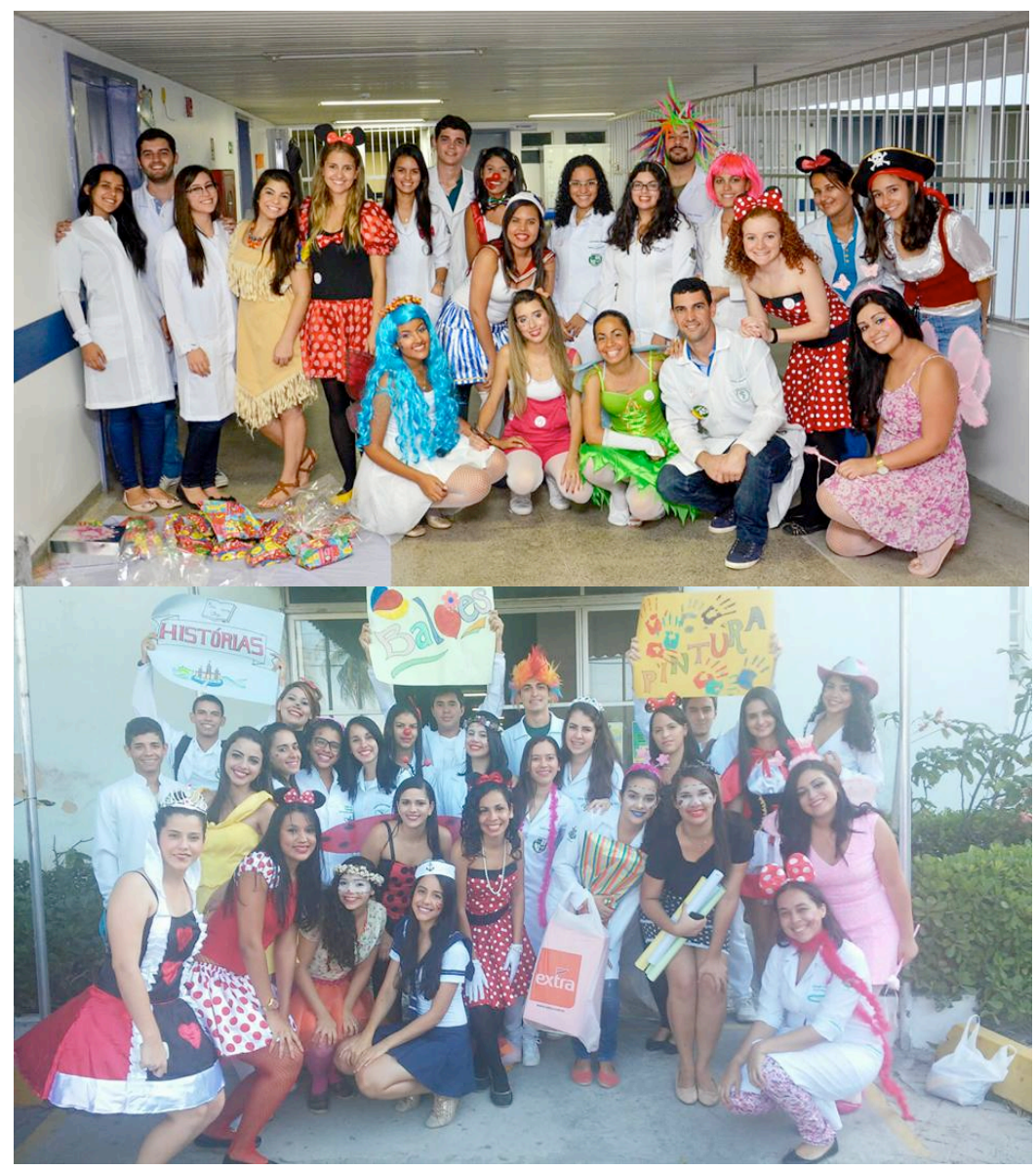

Figura 1: Registro fotográfico de Monitores do Acolher em ação alusiva ao Dia das Crianças. Fonte: Acervo do Projeto. Fotos autorizadas (2017).

De forma complementar, e com a finalidade de cumprir os objetivos das ações, são aproveitados brinquedos, fantoches, cartilhas e objetos diversos, advindos de doções ou de aquisições feitas pelos membros do Projeto no comércio local. Além desses recursos, a incorporação de personagens infantis, a contação de histórias, a utilização de atividades teatrais, dinâmicas, brincadeiras, apresentações artísticas e o emprego de músicas e poemas também contribuem para a quebra do paradigma destacado por Silva e Avelar (2007), que descreve o relacionamento do profissional de saúde com o paciente, por vezes, muito caracterizado como técnico, impessoal e com pouco envolvimento afetivo. Tais mecanismos são utilizados ainda com o objetivo de instigar a criança a interagir com a equipe do projeto e como forma de propor alternativas à realidade descrita por Chiattone (2003): 
Extensão universitária como ferramenta de promoção de saúde

Uma equipe de saúde extremamente profissional que não consegue visualizar a criança como um todo doente, que não consegue ampliar seu campo de ação e assumir comportamentos e atitudes flexíveis e carinhosas é certamente uma equipe que terá pouco sucesso junto às crianças hospitalizadas. Além disso, pacientes hospitalizados em enfermarias pediátricas extremamente rigorosas e frias com certeza mostrarão maiores sequelas causadas pela hospitalização (CHIATTONE, 2003, p. 30).

A abordagem do Projeto Acolher é realizada de forma a suavizar as rotinas e a minimizar a ausência do mundo exterior ao ambiente hospitalar a que ficam submetidas às crianças e seus acompanhantes, estando em clara harmonia com Política Nacional de Humanização (2013), que traz em sua descrição a importância da adoção de novas formas de exercer o cuidado, centradas no outro e introduzindo o afeto como forma de acolhimento.

Nas Figuras 1 e 2 observa-se, a equipe do Acolher em uma visita alusiva ao Dia da Criança e integrantes do Projeto no desenvolvimento de suas atividades regulares, respectivamente.

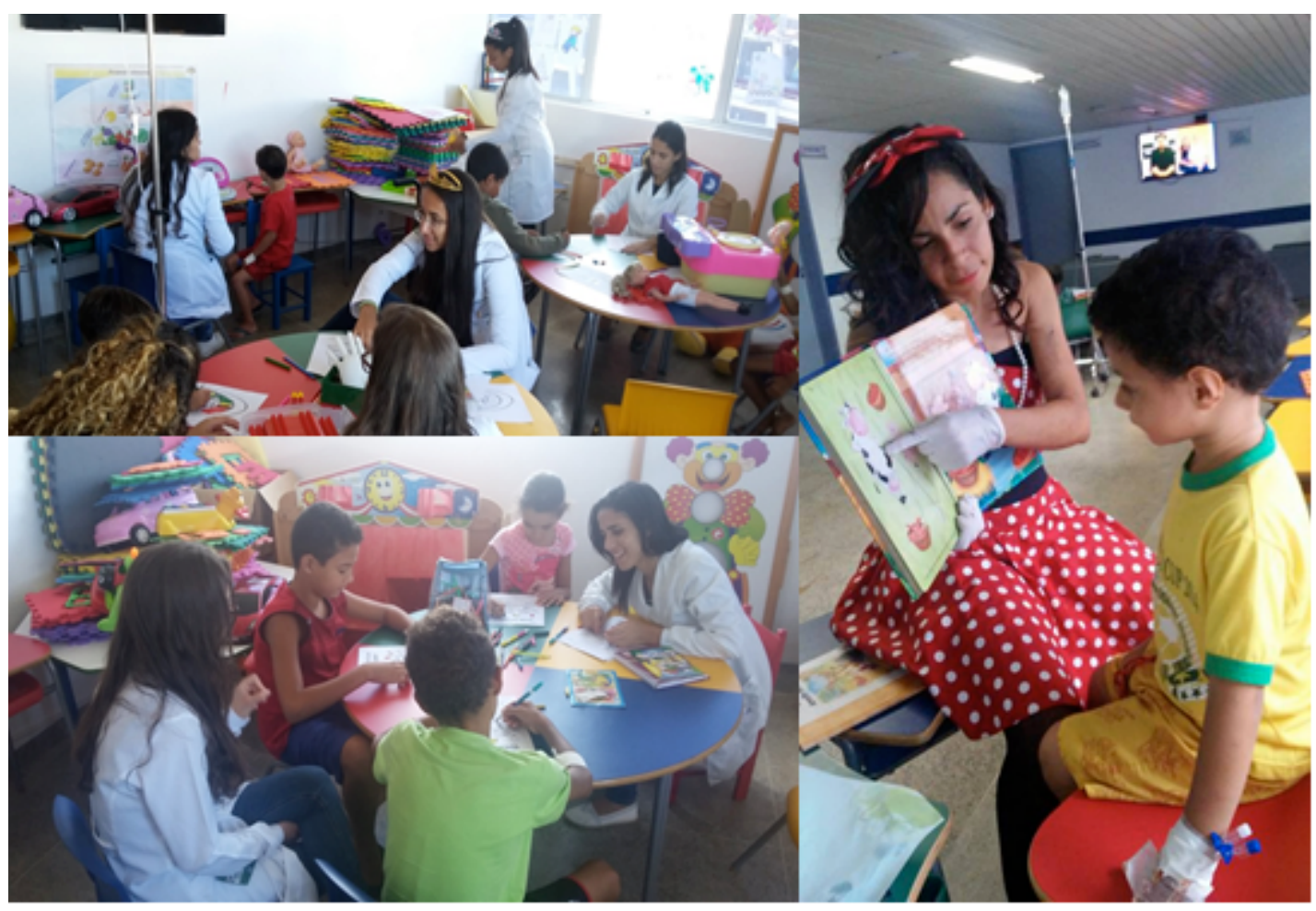

Figura 2: Registro fotográfico de Monitores do Acolher em ações regulares do Projeto.

Fonte: Acervo do Projeto. Fotos autorizadas (2017).

O Acolher insere-se no conjunto de ações que visam colocar em prática o que atualmente na saúde pública é conceituado como "reorientação das práticas de atenção 
à saúde", tendência marcada pela superação dos modelos demasiadamente centrados na doença, na assistência curativa e na via medicamentosa (SMEKE, 2002). Vislumbra-se com o Projeto introduzir práticas de atenção orientadas ativamente na construção de um "novo hospital", com um olhar que perpassa as atividades de cura e de reabilitação, que considera esse espaço como um instrumento de promoção de saúde, de defesa da vida, da cidadania e de ambiente para construção de inovações nas relações entre os serviços de saúde, seus profissionais e os usuários (ROLLO, 2006).

Nas visitas, busca-se diminuir a tensão imposta pelo meio hospitalar, possibilitando a criança reviver situações do seu cotidiano - como o ato de brincar, cantar e de ouvir histórias em quadrinhos. As atividades proporcionadas são para além de ações de saúde, constituindo fontes de prazer e fazendo com que, por vezes, a criança esqueça o seu sofrimento - mesmo que temporariamente - e vivencie momentos de descontração, fundamentais para sua qualidade de vida e para o processo de recuperação.

Sobre esse tema Smeke (2002) evidencia a necessidade de as práticas desenvolvidas serem reorientadas de modo que os modelos de atenção excessivamente centrados na doença, na assistência curativa e na intervenção medicamentosa, sejam superados em favor de outros orientados em direção à promoção da saúde, como as práticas de educação em saúde que buscam a qualidade de vida. 0 autor fala ainda da necessidade de capacitar a comunidade para que esta atue em prol da melhoria e da promoção da sua própria qualidade de vida e saúde.

As ações do Acolher estão em forte sintonia com a nova perspectiva de ambiente hospitalar proposta por Silva et al. (2009) que afirma ser o hospital "um território virgem de trabalho na perspectiva de humanização, do atendimento e da defesa da vida, sendo possível nesse espaço pensar em promoção da saúde, em educação em saúde e em criação de vínculos".

Como grande parte do tratamento hospitalar é inegavelmente imposto, a equipe do Projeto busca deixar os pacientes e seus acompanhantes livres para escolherem participarem ou não das atividades. Tal conduta é discutida por Cunha (2007) que afirma que "o acolhimento se caracteriza pela aceitação, compreensão e a capacidade de comunicação". Para o autor, a aceitação é demonstrada através da hospitalidade, do interesse, do apreço e na emissão de sentimentos sinceros. 
Extensão universitária como ferramenta de promoção de saúde

A avalição das atividades do Acolher é efetuada durante todo o curso do Projeto, adotando como principal instrumento um diário de bordo, em que o grupo de monitores do horário é responsável por descrever as experiências vivenciadas e relatar os sentimentos do grupo, frases marcantes, atitudes observadas e a receptividade dos pacientes, acompanhantes e profissionais. Em linhas gerais, a cada nova visita, observase uma boa integração entre as crianças, seus familiares e a equipe de monitores, sendo muito comum a participação ativa dos pais e acompanhantes, expressa principalmente por meio de questionamentos e de vivências pessoais por eles relatadas.

Cabe desatacar que cada ciclo do Projeto Acolher tem duração de um ano e, ao final desse período, os participantes assíduos e com frequência superior a $75 \%$ são certificados como monitores de extensão, variando a carga horária de acordo com a frequência de participação do voluntário. 0 acadêmico interessado em permanecer no projeto pode renovar seu vínculo para os ciclos posteriores, desde que tenha demostrado compromisso, frequência regular às visitas e não tenha recebido advertência por comportamento inapropriado ou falta injustificada às ações.

\section{Considerações finais}

O Projeto Acolher parte da premissa de que deve haver uma preocupação com a saúde global do paciente e também de seus familiares, de modo que considera que a criança e seus acompanhantes devem encontrar no Projeto e em suas ações um espaço de apoio terapêutico. Compreendemos que o brincar, assim como o educar, são direitos essenciais na vida da criança, que devem ser assegurados mesmo na condição de hospitalização, devendo tais ser introduzidos na rotina hospitalar enquanto parte integrante da assistência.

Através da experiência do Projeto, pode-se perceber a importância da humanização e de um atendimento pautado em acolhimento nas unidades de internação pediátrica. A partir da introdução do lúdico aliado à educação em saúde, pode-se propor alternativas às privações e restrições impostas pelo ambiente hospitalar, diminuindo a tensão do processo de internação e colocando em prática a promoção de saúde através de momentos de descontração, integração e de compartilhamento de conhecimentos.

Ademais, a partir das ações ao Acolher, seus membros podem trabalhar a melhoria das habilidades de comunicação, a capacidade de desenvolver atividades em 
Extensão universitária como ferramenta de promoção de saúde

grupo e aspectos como empatia, criação de vínculo, acolhimento e cuidado com o outro, além de colocar em prática o diálogo com profissionais, pacientes, familiares e acompanhantes.

Dessa forma, destacamos que as atividades desenvolvidas pelos monitores do Projeto Acolher, na medida em que visam promover a educação em saúde para pacientes pediátricos e seus familiares com a utilização de estratégias lúdicas, representam uma eficiente estratégia de compartilhamento de saberes e de geração de um ambiente mais acolhedor e humanizado, sendo que, aliadas, tais contribuem para a ressignificação da doença por parte dos sujeitos e para a promoção de saúde.

\section{Referências}

ANGERAMI-CAMON, V. A. O psicólogo no hospital. In: ANGERAMI-CAMON, V. A. (org.) Psicologia hospitalar: teoria e prática. São Paulo: Pioneira, 1995. p 1-14.

CHIATTONE, H. B. C. A criança e a hospitalização. In: ANGERAMI-CAMON, V. A. (org.) Psicologia hospitalar: a atuação do psicólogo no contexto hospitalar. 2. ed. São Paulo: Thomson, 2003. p. 23-100.

CRUZ, D. S. M.; COSTA, S. F. G.; NÓBREGA, M. L. Assistência Humanizada À Criança Hospitalizada. Rev. RENE. v. 7, n. 3, p. 98-104, 2006. Disponível em: <www.redalyc.org/pdf/3240/324027955014.pdf>. Acesso em: 9 jul. 2017.

CUNHA, N. H. O significado da Brinquedoteca Hospitalar. In: VEIGAS, D. Brinquedoteca Hospitalar: isto é humanização. 2. ed. Rio de Janeiro: Wared, 2007. p. 63-70.

GIL, A. C. Como elaborar projetos de pesquisa. 4. ed. São Paulo: Atlas, 2008.

GUIMARÃES, S. S. A hospitalização na infância. Psicologia: Teoria e pesquisa, v. 4, n. 2 , p. 102-112, 1998.1 Disponível em: <https://revistaptp.unb.br/index.php/ptp/article/view/1294/333>. Acesso em: 12 jul. 2017.

LUCHETTI, A. J.; MOREALE, V. C.; PARRO, M. C. Educação em saúde: uma experiência com teatro de fantoches no ensino nutricional de escolares. CuidArte. Enferm., v. 5, n. 2, p. 97-103, 2011. Disponível em: <http://saudepublica.bvs.br/pesquisa/resource/pt/bde22454>. Acesso em: 13 jul. 2017.

MERLEAU-PONTY, M. Fenomenologia da percepção. 2. ed. São Paulo: Martins Fontes, 1999.

MINISTÉRIO DA SAÚDE (Brasil). Política Nacional de Humanização. Brasília: Ministério da Saúde, 2013. 
MITRE, R M. A. et al. Promoção do brincar no contexto da hospitalização infantil como ação de saúde. Ciência \& Saúde coletiva, v. 9, n. 1, p. 147-154, 2004. Disponível em: <www.scielo.br/pdf/csc/v9n1/19832.pdf>. Acesso em: 12 jul. 2017.

PERIÓDICOS ELETRÔNICOS DE PSICOLOGIA. Sugestões para os relatos de experiência profissionais. 2014. Disponível em: <http://pepsic.bvsalud.org/>. Acesso em: 10 jul. 2017.

ROLLO, A. A. É possível construir novas práticas assistenciais no hospital público? In: MERHY, E. E.; ONOCKO R. (Org.) Agir em saúde: um desafio para o público. São Paulo: Hucitec, 2006.

SAGGESE, E. S. R.; MACIEL, M. DE A. Projeto saúde e brincar. Rio de Janeiro: Instituto Femandez Figueira - Fundação Oswaldo Cruz, 1997.

SILVA, A. M.; AVELAR, M. C. Q. O Acompanhante do Paciente Adulto Hospitalizado: percepção dos enfermeiros: uma abordagem qualitativa. Online Brasilian Journal of Nursing, v. 6, n. 3, 2007. Disponível em: <http://wwwdx.doi.org/10.5935/16764285.20071192>. Acesso em: 01 jul. 2017.

SILVA, M. A. M. et al. Promoção da saúde em ambientes hospitalares. In: Congresso brasileiro de Enfermagem, 61, Fortaleza, 2009. Disponível em: <http://www.abeneventos.com.br/anais_61cben/files/00324.pdf>. Acesso em: 06 jul. 2017.

SMEKE, E. L. M. Educação popular em saúde e formação profissional. Rev. de Atenção Primária à Saúde, v. 4, n. 9, p. 25-32, 2002.

VYGOTSKI, L. S. A formação social da mente. 4. ed. São Paulo: Martins Fontes, 1991. 
Extensão universitária como ferramenta de promoção de saúde

\section{University extension as a tool for health promotion and re-signification of hospitalized child care}

\begin{abstract}
This work describes the experience of the actions developed by the University Extension Project "Acolher", from the State University of Health Sciences of Alagoas (Uncisal), during the year 2016. The interventions were based on the development of educational and leisure activities in the pediatric health area in the Infant Care Nurseries at the State General Hospital in Maceió-AL, through the use of ludoterapia and health education. From the introduction of such strategies, it is possible to propose alternatives to the deprivations and restrictions imposed by the hospital environment, reducing the tension of the hospitalization process and putting into practice the health promotion through moments of relaxation, integration and sharing of knowledge.
\end{abstract}

Keywords: University Extension; Health education; Ludoterapia.

Original submetido em: 12 jul. 2017

Aceito para publicação em: 27 dez. 2017
Extensión universitaria como herramienta de promoción de salud y re significación del cuidado al niño hospitalizado

Resumen: Este trabajo describe la experiencia de las acciones desarrolladas por el Proyecto de Extensión Universitaria "Acolher", de la Universidad Estatal de Ciencias de la Salud de Alagoas (Uncisal), durante el año 2016. Las intervenciones realizadas fueron pautadas en el desarrollo de actividades lúdico-educativas en el área de salud pediátrica en las Enfermerías de cuidado infantil del Hospital General del Estado, en Maceió-AL, a través del empleo de la ludoterapia y de la educación en salud. A partir de la introducción de tales estrategias, se pueden proponer alternativas a las privaciones $\mathrm{y}$ restricciones impuestas por el ambiente hospitalario, disminuyendo la tensión del proceso de internación y poniendo en práctica la promoción de la salud a través de momentos de relajación, integración y de intercambio de conocimientos.

Palabras clave: Extensión Universitaria; Educación en Salud; Ludoterapia.

Sobre os autores:

\section{Claudio José dos Santos Júnior}

Estudante de Medicina da Universidade Estadual de Ciências da Saúde de Alagoas.

\section{Jailton Rocha Misael}

Enfermeiro Especialista em Urgência e Emergência. Acadêmico de Medicina, Universidade Estadual de Ciência da Saúde de Alagoas (UNCISAL). Colaborador do Projeto de Extensão “Acolher” (Uncisal).

\section{Raquel de Lima Chicuta}

Estudante de Nutrição, Universidade Federal de Alagoas (Ufal). Colaborador do Projeto de Extensão "Acolher" (Uncisal).

\section{Maria Rosa da Silva}

Enfermeira. Especialista Pediatria e Neonatologia pela Faculdade Integrada de Patos (FIP). Atualmente é Coordenadora Docente do 
Extensão universitária como ferramenta de promoção de saúde

Programa Pró-criança (Uncisal), co-orientadora do Projeto de Extensão Acolher (Uncisal) e orietandora do Projeto Sorriso de Plantão (Ufal).

\section{Claudio Fernando Rodrigues Soriano}

Médico Pediatra. Possui Mestrado e Doutorado em Medicina, ambos pela Faculdade de Medicina de Ribeirão Preto da Universidade de São Paulo (FMRP-USP). Atualmente é orientador do Projeto de Extensão Acolher (Uncisal) e co-orietandor do Projeto Sorriso de Plantão (Ufal).

\section{John Victor dos Santos Silva}

Estudante de Enfermagem, UNCISAL Monitor do Projeto de Extensão "Acolher" (Uncisal). 